Port Acadie

Revue interdisciplinaire en études acadiennes

An Interdisciplinary Review in Acadian Studies

porttcadie

\title{
L’édition critique en Acadie
}

\section{Denis Bourque et David Décarie}

Numéro 20-21, automne 2011, printemps 2012

L'édition critique et le développement du patrimoine littéraire en Acadie et dans les petites littératures

URI : https://id.erudit.org/iderudit/1010320ar

DOI : https://doi.org/10.7202/1010320ar

Aller au sommaire du numéro

Éditeur(s)

Université Sainte-Anne

ISSN

1498-7651 (imprimé)

1916-7334 (numérique)

Découvrir la revue

Citer ce document

Bourque, D. \& Décarie, D. (2011). L'édition critique en Acadie. Port Acadie, (20-21), 7-13. https://doi.org/10.7202/1010320ar d'utilisation que vous pouvez consulter en ligne. 


\section{L'édition critique en Acadie ${ }^{1}$}

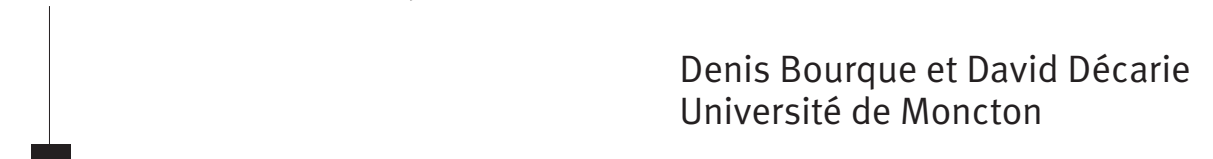

L'attribution du prix Goncourt à l'auteure acadienne Antonine Maillet en 1979 pour un roman (Pélagie-la-Charrette ${ }^{2}$ ) qui portait sur la déportation et le retour des Acadiens a suscité au Canada et même à travers le monde francophone un intérêt pour l'Acadie et sa littérature. À cette époque, Marguerite Maillet publiait aussi une Histoire de la littérature acadienne ${ }^{3}$, qui démontrait l'existence de cette littérature et la faisait remonter aux premiers jours de l'Acadie et de la Nouvelle-France, au moyen de nombreux récits de voyage portant sur la colonie acadienne naissante. Des travaux récents, comme la Bibliographie des publications d'Acadie 1609-1990 de Marguerite Maillet ${ }^{4}$ et Paroles d'Acadie - Anthologie de la littérature acadienne (1958-2009) de David Lonergan ${ }^{5}$, ont démontré que, loin de s'essouffler, la production littéraire acadienne a connu une véritable effervescence depuis sa création et, particulièrement, depuis les années 1970. Des centaines de livres ont été publiés : pièces de théâtre, romans, essais et, surtout, recueils de poésie. Plusieurs de ces œuvres, de même qu'un certain nombre d'ouvrages parus avant 1958 , se sont démarquées des autres et ont été consacrées par la critique universitaire au moyen de nombreuses thèses réalisées au Canada et dans divers pays étrangers, de nombreux comptes rendus et chapitres d'ouvrages collec-

1. Nous désirons remercier notre collègue Chantal Richard qui a aussi contribué à la rédaction de ce texte.

2. Antonine Maillet, Pélagie-la-Charrette, Montréal, Leméac, 1979.

3. Marguerite Maillet, Histoire de la littérature acadienne. De rêve en rêve, Moncton, Éditions d'Acadie, 1983.

4. Marguerite Maillet, Bibliographie des publications d'Acadie 1609-199o-Sources premières et sources secondes, Moncton, Chaire d'études acadiennes, Université de Moncton, 1992.

5. David Lonergan, Paroles d'Acadie - Anthologie de la littérature acadienne (19582009), Sudbury, Éditions Prise de parole, 2010. 
tifs, et surtout au moyen d'articles savants parus dans des revues prestigieuses au Canada, aux États-Unis et en Europe. Or il est aujourd'hui difficile de se procurer certaines de ces œuvres, en raison de la mince diffusion dont elles font l'objet au Canada et à l'étranger.

Ce phénomène s'explique en partie par la fragilité de l'édition en Acadie, dont témoigne la disparition en 2001 des Éditions d'Acadie, qui occupait une place importante dans la diffusion des œuvres acadiennes. La fermeture des Éditions d'Acadie a été désastreuse pour la littérature acadienne, puisqu'elle a retiré de la circulation un nombre considérable d'œuvres marquantes. Dispersée maintenant dans de petites maisons d'édition trop souvent éphémères et devant travailler avec peu de moyens, la littérature acadienne peine à se constituer en un corpus national. Pourtant, la littérature acadienne continue de susciter de l'intérêt chez les chercheurs au pays et à l'étranger, qui bénéficieront bientôt, grâce au travail d'une équipe de l'Université de Moncton, d'un Dictionnaire des cuvres littéraires de l'Acadie des Maritimes du xx siècle ${ }^{6}$,mais l'accès à une édition de qualité de ces œuvres, voire à une édition tout court, demeure problématique. Dans un grand nombre de cas, celles-ci sont inaccessibles au public, car elles sont inédites pour certaines, conservées dans des bibliothèques ou dépôts d'archives pour d'autres ou, ce qui est bien souvent le cas, tout simplement épuisées.

La solution pour lutter contre cette inaccessibilité et cette fragmentation de la littérature acadienne a paru évidente à un groupe de professeurs affiliés surtout à l'Université de Moncton : il s'agissait de rassembler les grands textes acadiens au sein d'une collection, à l'instar des collections du «Nénuphar » (Fides) et plus récemment de la « Bibliothèque du Nouveau Monde » (BNM). Celles-ci ont joué et jouent encore un rôle de premier plan dans l'autonomisation et le rayonnement de la littérature québécoise. En Acadie, Marguerite Maillet, alors qu'elle était titulaire de la Chaire d'études acadiennes à l'Université de Moncton, avait déjà, au début des années quatre-vingt-dix, formé un groupe de chercheurs dans le but de publier, sous forme d'éditions critiques, les textes importants de la littérature acadienne. À la suite de ces efforts, deux titres ont été publiés ${ }^{7}$, mais le projet a dû être abandonné, faute de moyens. Le Groupe de recherche en édition critique de l'Université de Moncton (GrÉcum), fondé

6. Ce dictionnaire, sous la direction de Janine Gallant et Maurice Raymond de l'Université de Moncton, doit paraître en 2012 aux Éditions Prise de parole.

7. Pascal Poirier, Causerie memramcookienne (édition critique établie par Pierre M. Gérin), Moncton, Chaire d'études acadiennes, Université de Moncton, 1990 et André-T. Bourque, Chez les anciens Acadiens - Causeries du grand-père Antoine, édition critique établie par Lauraine Léger, Moncton, Chaire d'études acadiennes, Université de Moncton, 1994. 
en 2004, se situe d'emblée dans la continuité de ce travail de pionnier entrepris par M. Maillet et s'est donné comme tâche principale la constitution d'une collection qui rassemblera, sous forme d'éditions critiques, les textes fondamentaux de la littérature acadienne, des origines à nos jours.

Notre collection permettra de réaliser six objectifs généraux, soit : 1) consolider la mémoire littéraire commune de l'Acadie; 2) assurer la pérennité de la littérature acadienne; 3) faciliter l'enseignement de la littérature acadienne en fournissant aux professeurs des éditions sûres et de qualité, alors que de très nombreux titres sont inédits, épuisés et inaccessibles pour les étudiants; 4) stimuler les recherches sur la littérature acadienne en Acadie, au Canada et à l'étranger; 5) former de jeunes chercheurs afin qu'ils puissent entreprendre des thèses en édition critique et assurer ainsi une relève sur le plan de l'édition critique en Acadie; 6) favoriser la diffusion de la littérature acadienne en Acadie, au Québec, au Canada et à l'étranger ${ }^{8}$.

Nos éditions critiques viennent combler un vide au niveau de la recherche et de l'enseignement universitaire en études acadiennes. Elles contribueront à enrichir considérablement cette recherche et les cours qui se donnent dans le domaine. Ainsi, nous comptons pouvoir rejoindre avec nos livres l'immense marché des universités nord-américaines et européennes, où l'intérêt, voire l'engouement, pour les littératures francophones, y compris la littérature acadienne, ne cesse de grandir. Or nous sommes convaincus que la diffusion de nos éditions critiques débordera, et largement, le milieu universitaire auquel elles sont d'abord destinées. Car la pénurie d'ouvrages acadiens disponibles qui affecte actuellement les milieux universitaires se fait aussi sentir de façon aiguë dans les écoles acadiennes. Nous procurerons aux enseignants du secondaire des textes fiables et bien établis, accompagnés d'une quantité très importante de renseignements qui faciliteront pour eux l'enseignement de la littérature acadienne. Et il est sûr que nos éditions critiques intéresseront un public encore plus étendu de lecteurs, qu'ils soient dans les provinces des Maritimes, ailleurs au Canada ou à l'étranger. Nos livres, nos communications, nos articles susciteront sans aucun doute un nouvel essor des études acadiennes et constitueront un apport important à l'étude de la littérature canadienne et francophone. Nous comptons utiliser les nouvelles technologies de linformation pour promouvoir nos recherches. En 2012,

8. Voir David Décarie, "L'édition des textes fondamentaux de la littérature acadienne ", dans Jacinthe Martel (dir.), Archives littéraires et manuscrits d'écrivains - Politiques et usages du patrimoine, Québec, Éditions Nota bene, coll. "Convergences », 2008, p. 145-154. 
nous préparerons un nouveau site Web, qui fera état des activités de notre groupe de recherche et qui présentera nos éditions critiques publiées ou en cours de réalisation. Le site aura un volet interactif et offrira au public la possibilité de commander nos livres. Nous envisageons également la publication sur ce site des travaux de recherche de nos membres, de documents visuels, sonores ou audiovisuels qui accompagnent ces travaux, ainsi que de certaines éditions critiques en cours ou achevées. Des éditions critiques seront éventuellement disponibles sur cédérom.

L'équipe est formée surtout de professeurs chevronnés, dont la plupart possèdent une expérience considérable dans le domaine de l'édition critique ou des études acadiennes. En tout, onze chercheurs composent le Groupe de recherche en édition critique : sept professeurs du Département d'études françaises de l'Université de Moncton (Denis Bourque, Raoul Boudreau, David Décarie, Pierre Gérin, Ronald Labelle, Jean Morency et Maurice Raymond), une professeure de l'Université du Nouveau-Brunswick (Chantal Richard), une professeure de l'Université Mount Allison (Monika Boehringer), un professeur de l'Université SainteAnne (Jimmy Thibeault) et un chargé de cours de l'Université de Paris iv et de la Maison des sciences de l'Homme de Paris (Bernard Émont). Ainsi les trois principales universités du Nouveau-Brunswick sont représentées au sein de l'équipe, ainsi que la seule autre université francophone des provinces de l'Atlantique, avec l'Université de Moncton. Denis Bourque poursuit l'édition critique de deux ouvrages : Les Crasseux $(1968,1973,1974)$, que l'on peut considérer comme la pièce fondatrice de l'œuvre d'Antonine Maillet ${ }^{9}$, et Les Entretiens du village $(1957,1979)$ d’Emery LeBlanc ${ }^{10}$, recueil qui signale de façon remarquable la fin de la littérature acadienne traditionnelle; Pierre Gérin et Bernard Émont réalisent une édition critique du texte fondateur de la littérature acadienne : l'Histoire de la NouvelleFrance (1609) de Marc Lescarbot ${ }^{11}$; Ronald Labelle, une édition critique d'un livre controversé à l'époque de sa parution, L'École aux apparitions mystérieuses (1896) de Philéas-F. Bourgeois ${ }^{12}$; David Décarie, une édition

9. Antonine Maillet, Les Crasseux, Montréal, Holt, Rinehart et Winston Ltée; Montréal, Leméac, 1973; nouvelle version revue et considérablement augmentée pour la scène, Montréal, Leméac, 1974.

10. Emery Leblanc, Les Entretiens du village, Moncton, Imprimerie acadienne, 1957; nouvelle édition revue et corrigée, Moncton, Éditions d’Acadie, 1979.

11. Marc Lescarbot, Histoire de la Nouvelle France. Contenant les navigations, découvertes, \& habitations faites par les François ès Indes Occidentales \& Nouvelle France souz l'avoeu et authorité de noz Rois Tres-Chrétiens, \& les diverses fortunes d'iceux en l'execution de ces choses, depuis cent ans jusques à hui - En quoy est comprise l'Histoire Morale, Naturelle, et Geographique de ladite province; Avec les Tables \& Figures d'icelle, Paris, Chez Jean Milot, 1609.

12. Philias-F. Bourgeois, L'École aux apparitions mystérieuses, Montréal, C.O. 
critique de l'un des premiers et des plus importants romans acadiens, Les Portes tournantes (1984) de Jacques Savoie ${ }^{13}$; Raoul Boudreau, une édition critique de Mourir à Scoudouc (1974) d'Herménégilde Chiasson ${ }^{14}$, recueil qui a marqué de façon incontournable la poésie acadienne contemporaine; Chantal Richard, une édition critique de Bloupe (1994) de Jean Babineau ${ }^{15}$, auteur qui a contribué au renouvellement de la forme romanesque en Acadie; Monika Boehringer, une édition critique de deux des premiers romans de l'écrivaine contemporaine la plus remarquée, France Daigle - Film d'amour et de dépendance $(1984)^{16}$ et Histoire de la maison qui brûle (1985) ${ }^{17}$; Jean Morency, une édition critique du poème Évangéline (1847) de Henry Wadsworth Longfellow traduit par Pamphile Le May (1865, $1870,1912)^{18}$, œuvre qui, peut-être plus que toutes les autres, a marqué l'Acadie et sa littérature; Jimmy Thibeault, une édition critique d'un des premiers romans acadiens, Le Chef des Acadiens $(1956,1980)$ de l'écrivain néo-écossais Alphonse Deveau ${ }^{19}$, premier texte littéraire à rompre avec l'image traditionnelle des Acadiens perçus comme peuple marty ${ }^{20}$; et Maurice Raymond, une édition critique de trois recueils de poésie de Ronald Després qui, avec les premiers ouvrages d'Antonine Maillet, ont fondé la modernité acadienne : Silences à nourrir de sang $(1958)^{21}$, Les Cloisons en vertige (1962) ${ }^{22}$, Le Balcon des dieux inachevés (1968) ${ }^{23}$.

Notre groupe a bénéficié d'une importante subvention du CRSH pour 2007-2010, qui a permis de réaliser un travail de recherche considérable en vue de la publication de plusieurs éditions critiques. Outre les ouvrages énumérés ci-dessus, trois titres ont été acceptés pour publication par notre éditeur, l'Institut de recherches en études acadiennes de l'Université de Moncton, dans une nouvelle collection consacrée à nos éditions cri-

\section{Beauchemin et Fils, 1896.}

13. Jacques Savoie, Les Portes tournantes, Montréal, Boréal Express, 1984.

14. Herménégilde Chiasson, Mourir à Scoudouc, Moncton, Éditions d’Acadie, 1974.

15. Jean Babineau, Bloupe, Moncton, Éditions Perce-Neige, 1993.

16. France Daigle, Film d'amour et de dépendance - Chef-d'œuvre obscur, Moncton, Éditions d'Acadie, 1984.

17. France Daigle, Histoire de la maison qui brûle-Vaguement suivi d'un dernier regard sur la maison qui brûle, Moncton, Éditions d'Acadie, 1985.

18. Pamphile Le May, Essais poétiques, Québec, G. E. Desbarats, Imprimeur-Éditeur, 1865; Évangéline, traduction du poème acadien, Québec, P.-G. Delisle, 1870; Évangéline et autres poèmes de Longfellow, traduction libre par Pamphile Le May, Montréal, J.-Alfred Guay, 1912.

19. J.-Alphonse Deveau, Le Chefdes Acadiens, Yarmouth, J. A. Hamon, 1956; $2^{\mathrm{e}}$ édition révisée, Yarmouth (N.-É.), Éditions Lescarbot, 1980.

20. Voir à ce sujet Maillet, Histoire de la littérature acadienne, op. cit., p. 159.

21. Ronald Després, Silences à nourrir de sang, Montréal, Éditions d’Orphée, 1958.

22. Ronald Després, Les Cloisons en vertige, Montréal, Beauchemin, 1962.

23. Ronald Després, Le Balcon des dieux inachevés, Québec, Éditions Garneau, 1968. 
tiques intitulée "Bibliothèque acadienne " : de Denis Bourque et Chantal Richard, coéditeurs, l'Édition critique des sermons et discours des grandes conventions nationales acadiennes (Memramcook 1881, Miscouche 1884, Pointe-de-l'Église 1890, Arichat 1900, Caraquet 1905, Saint-Basile 1908, Grand Pré et Pointe-de-l'Église 1921, Moncton 1927, Memramcook 1937), en trois volumes ${ }^{24}$; de Chantal Richard, l'édition critique de Poèmes acadiens de Napoléon Landry 25 et, de Monika Boehringer, l'édition critique de Sans jamais parler du vent de France Daigle ${ }^{26}$. La publication de ces ouvrages est prévue en 2012. En 2012 également, Pierre Gérin prévoit soumettre pour publication son édition critique de Subercase d'Alexandre Braud $(1902,1936)^{27}$ et Ronald Labelle son édition critique de Contes acadiens de Thomas LeBlanc ${ }^{28}$. Le travail accompli jusqu'à maintenant a également permis la publication d'articles et de chapitres de livres par les membres du Groupe de recherche et la participation à divers colloques, dont un colloque important, financé par le CRSH et l'Université de Moncton.

Organisé par Denis Bourque, David Décarie et Pierre Gérin en août 2010 à l'Université de Moncton et intitulé « L'édition critique et le développement du patrimoine littéraire en Acadie et dans les petites littératures », ce colloque réunissait vingt chercheurs de l'Acadie, du Québec, de France et des États-Unis. Quatorze des vingt communications ont été transformées en articles, qui se retrouvent dans ces deux numéros spéciaux de la revue Port Acadie. Ainsi le lecteur retrouvera entre ces pages les principaux résultats des travaux des chercheurs membres du Groupe de recherche en édition critique et de leurs étudiants, en plus d'articles fournis par des collaborateurs extérieurs portant sur les littératures acadienne, québécoise, louisianaise et de l'Ouest canadien. Complètent le dossier un article sur l'édition critique du premier tome de l'Histoire de la

24. Le premier volume contient une réédition partielle du livre Recueil des travaux et délibérations des six premières conventions compilé par Ferdinand J. Robidoux, vol. I, Memramcook, Miscouche, Pointe-de-l'Église, 1881, 1884, 1890, Shédiac, Impr. du Moniteur Acadien, 1907. Le second volume de ce recueil, annoncé dans le titre par Robidoux, n’a pas été publié.

25. Napoléon-P. Landry, Poèmes acadiens, Montréal [et] Paris, Fides, 1955.

26. France Daigle, Sans jamais parler du vent - Roman de crainte et d'espoir que la mort arrive à temps, Moncton, Éditions d'Acadie, 1983.

27. Alexandre Braud, Subercase ou les Dernières Années de la domination française en Acadie (Drame historique en trois actes et un épilogue), [paru dans le Moniteur acadien, en huit tranches, du 14 avril au 30 octobre 1902, l'auteur a, par la suite, modifié les $1^{\text {er }}$ et $2^{\mathrm{e}}$ actes et refait complètement le $3^{\mathrm{e}}$ acte et l'épilogue; manuscrit au Centre d'études acadiennes, fonds Ferdinand-J.-Robidoux, 4.6-14].

28. Inédit. 
Nouvelle-France de Marc Lescarbot et une interview portant sur l'édition critique électronique avec l'écrivain Jean Babineau, auteur de Bloupe. 